\title{
Highly Stereoselective Michael Addition Reactions of camTHP* Desymmetrised Glycinamide for the Synthesis of Functionally Dense Amino Acid Derivatives
}

\author{
Darren J. Dixon, ${ }^{a \star}$ Richard A. J. Horan, ${ }^{b}$ Nathaniel J. T. Monck ${ }^{c}$ \\ and Paul Berg \\ ${ }^{a}$ Department of Chemistry, University of Manchester, Manchester, M13 9PL, UK. \\ ${ }^{b}$ Department of Chemistry, University of Cambridge, Lensfield Road, Cambridge \\ CB2 1EW, UK.
}

${ }^{c}$ Vernalis, Oakdene Court, 613 Reading Road, Winnersh, Wokingham, RG41 5UA. Darren.Dixon@man.ac.uk

\section{Supporting Information}

Experimental (P S1-S5)

General procedure for the Michael reaction of 1<smiles>CN(C)C(=O)CN(C(=O)O)[C@H]1CCCC2(OC(=O)[O-])C1CCC2(C)C</smiles>

1

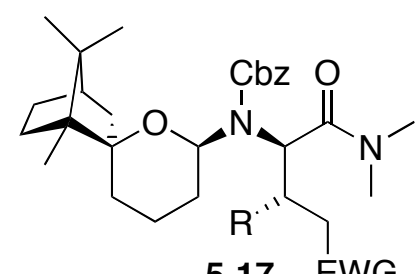

5-17 EWG

To a stirred solution of 1 (133 $\mathrm{mg}, 0.3 \mathrm{mmol})$ in degassed THF $(3 \mathrm{ml})$ at $-78{ }^{\circ} \mathrm{C}$ under argon was added LiHMDS ( $1.0 \mathrm{M}$ in THF, $0.6 \mathrm{ml}, 0.6 \mathrm{mmol})$ and the pale yellow solution stirred for 1 hour before the addition of Michael acceptor $(0.36 \mathrm{mmol}, 1.2 \mathrm{eq})$. The reaction was stirred at $-78{ }^{\circ} \mathrm{C}$ for 1 hour and at $-20^{\circ} \mathrm{C}$ for a further 1 hour before being quenched at $-20{ }^{\circ} \mathrm{C}$ by the rapid addition of $\mathrm{AcOH}(0.08 \mathrm{ml}, 1.2 \mathrm{mmol})$. After warming to room temperature the reaction mixture was filtered through a plug of silica $(10 \mathrm{~mm} \times 10 \mathrm{~mm}$ dia.) eluting with EtOAc $(50 \mathrm{ml})$ and concentrated in vacuo. The crude product was purified by column chromatography (silica, neat petroleum ether $\left(40-60^{\circ} \mathrm{C}\right.$ ) gradient to neat $\mathrm{Et}_{2} \mathrm{O}$ ) to give the products $5-17$.

Data for $5\left(\mathrm{R}=\mathrm{Ph}, \mathrm{EWG}=\mathrm{NO}_{2}\right)$

${ }^{1} \mathrm{H}-\mathrm{NMR}\left(\mathrm{d}_{6}\right.$-DMSO, $\left.120^{\circ} \mathrm{C}, 500 \mathrm{MHz}\right):$ 7.36-7.30 (m, 5H), 7.25-7.20 (m, 5H), $5.36(\mathrm{~d}, J=$ $9.6 \mathrm{~Hz}, 1 \mathrm{H}), 5.20(\mathrm{dd}, J=11.5,2.1 \mathrm{~Hz}, 1 \mathrm{H}), 5.03(\mathrm{~d}, J=12.3 \mathrm{~Hz}, 1 \mathrm{H}), 4.96(\mathrm{dd}, J=13.1$, $9.5 \mathrm{~Hz}, 1 \mathrm{H}$ ), 4.81 (d, $J=12.3 \mathrm{~Hz}, 1 \mathrm{H}), 4.77$ (dd, $J=13.1,4.0 \mathrm{~Hz}, 1 \mathrm{H}$ ), 4.33 (ddd, $J=9.6$, 9.5, 4.0 Hz, 1H), $2.92(\mathrm{~s}, 6 \mathrm{H}), 2.23(\mathrm{ddt}, J=12.6,11.8,4.1 \mathrm{~Hz}, 1 \mathrm{H}), 2.08(\mathrm{dt}, J=12.8,3.4$ $\mathrm{Hz}, 1 \mathrm{H}), 1.87-1.82(\mathrm{~m}, 1 \mathrm{H}), 1.67-1.26(\mathrm{~m}, 8 \mathrm{H}), 1.15(\mathrm{~d}, J=12.8,1 \mathrm{H}), 0.96-0.91(\mathrm{~m}, 1 \mathrm{H})$, $0.91(\mathrm{~s}, 3 \mathrm{H}), 0.82(\mathrm{~s}, 3 \mathrm{H}), 0.76(\mathrm{~s}, 3 \mathrm{H})$; HRMS $(\mathrm{ESI}+) \mathrm{m} / \mathrm{z}$ Found $[\mathrm{M}+\mathrm{H}]^{+}$592.3386, $\mathrm{C}_{34} \mathrm{H}_{46} \mathrm{~N}_{3} \mathrm{O}_{6}{ }^{+}$requires 592.3381 . 


\section{Data for $6\left(\mathrm{R}=2-\mathrm{Nap}, \mathrm{EWG}=\mathrm{NO}_{2}\right)$}

${ }^{1} \mathrm{H}-\mathrm{NMR}\left(\mathrm{d}_{6}\right.$-DMSO, $\left.120{ }^{\circ} \mathrm{C}, 500 \mathrm{MHz}\right): 7.87-7.80(\mathrm{~m}, 3 \mathrm{H}), 7.76(\mathrm{~d}, J=8.5 \mathrm{~Hz}, 1 \mathrm{H}), 7.54$ (dd, $J=8.5,1.8 \mathrm{~Hz}, 1 \mathrm{H}), 7.51-7.47(\mathrm{~m}, 2 \mathrm{H}), 7.28-7.19(\mathrm{~m}, 3 \mathrm{H}), 7.09(\mathrm{~d}, J=7.2 \mathrm{~Hz}, 2 \mathrm{H})$, $5.51(\mathrm{~d}, J=9.7 \mathrm{~Hz}, 1 \mathrm{H}), 5.18(\mathrm{dd}, J=11.5,2.0 \mathrm{~Hz}, 1 \mathrm{H}), 5.08(\mathrm{dd}, J=13.1,9.5 \mathrm{~Hz}, 1 \mathrm{H})$, $4.96(\mathrm{~d}, J=12.2 \mathrm{~Hz}, 1 \mathrm{H}), 4.87$ (dd, $J=13.1,4.0 \mathrm{~Hz}, 1 \mathrm{H}), 4.73(\mathrm{~d}, J=12.2 \mathrm{~Hz}, 1 \mathrm{H}), 4.53$ (ddd, $J=9.7,9.5,4.0 \mathrm{~Hz}, 1 \mathrm{H}$ ), $2.95(\mathrm{~s}, 6 \mathrm{H}), 2.29$ (ddt, $J=12.4,11.9,4.0 \mathrm{~Hz}, 1 \mathrm{H}$ ), 2.06 (dt, $J=12.7,3.3 \mathrm{~Hz}, 1 \mathrm{H}), 1.89-1.85(\mathrm{~m}, 1 \mathrm{H}), 1.73-1.23(\mathrm{~m}, 8 \mathrm{H}), 1.13(\mathrm{~d}, J=12.7,1 \mathrm{H}), 0.95-$ $0.90(\mathrm{~m}, 1 \mathrm{H}), 0.90(\mathrm{~s}, 3 \mathrm{H}), 0.82(\mathrm{~s}, 3 \mathrm{H}), 0.77(\mathrm{~s}, 3 \mathrm{H})$; HRMS (ESI+) $\mathrm{m} / z$ Found $[\mathrm{M}+\mathrm{H}]^{+}$ $642.3533, \mathrm{C}_{38} \mathrm{H}_{48} \mathrm{~N}_{3} \mathrm{O}_{6}^{+}$requires 642.3538.

\section{Data for 7 (R=PMP, EWG=NO}

${ }^{1} \mathrm{H}-\mathrm{NMR}\left(\mathrm{d}_{6}\right.$-DMSO, $\left.120^{\circ} \mathrm{C}, 500 \mathrm{MHz}\right):$ 7.36-7.31 (m, 3H), 7.25-7.22 (m, 4H), 6.80-6.77 $(\mathrm{m}, 2 \mathrm{H}), 5.30(\mathrm{~d}, J=9.7 \mathrm{~Hz}, 1 \mathrm{H}), 5.19(\mathrm{dd}, J=11.4,2.1 \mathrm{~Hz}, 1 \mathrm{H}), 5.06(\mathrm{~d}, J=12.3 \mathrm{~Hz}, 1 \mathrm{H})$, $4.89(\mathrm{dd}, J=13.0,9.5 \mathrm{~Hz}, 1 \mathrm{H}), 4.82(\mathrm{~d}, J=12.3 \mathrm{~Hz}, 1 \mathrm{H}), 4.72(\mathrm{dd}, J=13.0,4.0 \mathrm{~Hz}, 1 \mathrm{H})$, 4.26 (ddd, $J=9.7,9.5,4.0 \mathrm{~Hz}, 1 \mathrm{H}$ ), 3.75 (s, 3H), 2.91 (s, 6H), 2.20 (ddt, $J=12.6,11.8,4.0$ $\mathrm{Hz}, 1 \mathrm{H}), 2.08(\mathrm{dt}, J=13.2,4.0 \mathrm{~Hz}, 1 \mathrm{H}), 1.86-1.82(\mathrm{~m}, 1 \mathrm{H}), 1.64-1.25(\mathrm{~m}, 8 \mathrm{H}), 1.15(\mathrm{~d}, J=$ 12.8, 1H), 0.96-0.91 (m, 1H), $0.91(\mathrm{~s}, 3 \mathrm{H}), 0.82(\mathrm{~s}, 3 \mathrm{H}), 0.75(\mathrm{~s}, 3 \mathrm{H}) ; \mathrm{HRMS}(\mathrm{ESI}+) \mathrm{m} / \mathrm{z}$ Found $[\mathrm{M}+\mathrm{H}]^{+} 622.3490, \mathrm{C}_{35} \mathrm{H}_{48} \mathrm{~N}_{3} \mathrm{O}_{7}^{+}$requires 622.3487.

\section{Data for $8\left(\mathrm{R}={ }^{\mathrm{n}} \mathrm{Hexyl}, \mathrm{EWG}=\mathrm{NO}_{2}\right)$}

${ }^{1} \mathrm{H}-N M R\left(\mathrm{~d}_{6}\right.$-DMSO, $\left.120^{\circ} \mathrm{C}, 500 \mathrm{MHz}\right): 7.39-7.33(\mathrm{~m}, 5 \mathrm{H}), 5.18(\mathrm{~d}, J=12.3 \mathrm{~Hz}, 1 \mathrm{H}), 5.14$ (dd, $J=11.3,2.2 \mathrm{~Hz}, 1 \mathrm{H}), 5.10(\mathrm{~d}, J=12.3 \mathrm{~Hz}, 1 \mathrm{H}), 4.99(\mathrm{~d}, J=9.8 \mathrm{~Hz}, 1 \mathrm{H}), 4.57$ (dd, $J=$ 13.3, 5.4 Hz, 1H), $4.54(\mathrm{dd}, J=13.3,4.3 \mathrm{~Hz}, 1 \mathrm{H}), 2.88(\mathrm{~s}, 6 \mathrm{H}), 2.85-2.79(\mathrm{~m}, 1 \mathrm{H}), 2.12(\mathrm{dt}$, $J=12.5,3.5 \mathrm{~Hz}, 1 \mathrm{H}), 2.04(\mathrm{ddt}, J=12.4,12.0,4.1 \mathrm{~Hz}, 1 \mathrm{H}), 1.82-1.76(\mathrm{~m}, 1 \mathrm{H}), 1.68-1.20$ (m, 19H), $0.98(\mathrm{~s}, 3 \mathrm{H}), 0.97-0.93(\mathrm{~m}, 1 \mathrm{H}), 0.88(\mathrm{t}, J=7.1 \mathrm{~Hz}, 3 \mathrm{H}), 0.84(\mathrm{~s}, 3 \mathrm{H}), 0.74(\mathrm{~s}$, $3 \mathrm{H})$; HRMS (ESI+) $\mathrm{m} / \mathrm{z}$ Found $[\mathrm{M}+\mathrm{H}]^{+} 600.4009, \mathrm{C}_{34} \mathrm{H}_{54} \mathrm{~N}_{3} \mathrm{O}_{6}{ }^{+}$requires 600.4007.

\section{Data for $9\left(\mathrm{R}=\mathrm{i} \mathrm{Pr}, \mathrm{EWG}=\mathrm{NO}_{2}\right)$}

${ }^{1} \mathrm{H}-N M R\left(\mathrm{~d}_{6}\right.$-DMSO, $\left.120^{\circ} \mathrm{C}, 500 \mathrm{MHz}\right): 7.42-7.31(\mathrm{~m}, 5 \mathrm{H}), 5.17(\mathrm{~d}, J=12.3 \mathrm{~Hz}, 1 \mathrm{H}), 5.12$ $(\mathrm{d}, J=12.3 \mathrm{~Hz}, 1 \mathrm{H}), 5.08(\mathrm{dd}, J=11.3,2.1 \mathrm{~Hz}, 1 \mathrm{H}), 5.05(\mathrm{~d}, J=10.0 \mathrm{~Hz}, 1 \mathrm{H}), 4.53(\mathrm{dd}, J$ $=14.5,5.0 \mathrm{~Hz}, 1 \mathrm{H}), 4.29(\mathrm{dd}, J=14.5,4.3 \mathrm{~Hz}, 1 \mathrm{H}), 2.96-2.91(\mathrm{~m}, 1 \mathrm{H}), 2.89(\mathrm{~s}, 6 \mathrm{H}), 2.10$ (dt, $J=12.8,3.7 \mathrm{~Hz}, 1 \mathrm{H}$ ), 2.05 (ddt, $J=12.4,12.0,4.1 \mathrm{~Hz}, 1 \mathrm{H}$ ), 1.95 (double septet, $J=$ 6.9, $10.1 \mathrm{~Hz}, 1 \mathrm{H}), 1.80-1.76(\mathrm{~m}, 1 \mathrm{H}), 1.68-1.26(\mathrm{~m}, 8 \mathrm{H}), 1.24(\mathrm{~d}, J=12.8 \mathrm{~Hz}, 1 \mathrm{H}), 0.99(\mathrm{~s}$, $3 \mathrm{H}), 0.98-0.95(\mathrm{~m}, 1 \mathrm{H}), 0.94(\mathrm{~d}, J=6.9 \mathrm{~Hz}, 3 \mathrm{H}), 0.84(\mathrm{~s}, 3 \mathrm{H}), 0.83(\mathrm{~d}, J=6.9 \mathrm{~Hz}, 3 \mathrm{H}), 0.72$ $(\mathrm{s}, 3 \mathrm{H})$; HRMS (ESI+) $\mathrm{m} / \mathrm{z}$ Found $[\mathrm{M}+\mathrm{H}]^{+}$558.3541, $\mathrm{C}_{31} \mathrm{H}_{48} \mathrm{~N}_{3} \mathrm{O}_{6}{ }^{+}$requires 558.3538.

\section{Data for $10\left(\mathrm{R}=\mathrm{Me}, \mathrm{EWG}=\mathrm{CO}_{2}{ }^{\mathrm{t}} \mathrm{Bu}\right)$}

${ }^{1} \mathrm{H}-\mathrm{NMR}\left(\mathrm{d}_{6}\right.$-DMSO, $\left.120^{\circ} \mathrm{C}, 500 \mathrm{MHz}\right): 7.40-7.31(\mathrm{~m}, 5 \mathrm{H}), 5.16(\mathrm{~d}, J=12.4 \mathrm{~Hz}, 1 \mathrm{H}), 5.12$ $(\mathrm{d}, J=12.4 \mathrm{~Hz}, 1 \mathrm{H}), 5.04(\mathrm{dd}, J=11.2,2.2 \mathrm{~Hz}, 1 \mathrm{H}), 4.87(\mathrm{~d}, J=10.8 \mathrm{~Hz}, 1 \mathrm{H}), 2.89(\mathrm{~s}$, $6 \mathrm{H}), 2.63-2.54(\mathrm{~m}, 1 \mathrm{H}), 2.25(\mathrm{dd}, J=14.6,3.7 \mathrm{~Hz}, 1 \mathrm{H}), 2.17-2.09(\mathrm{~m}, 2 \mathrm{H}), 1.99(\mathrm{dd}, J=$ 14.6, $8.4 \mathrm{~Hz}, 1 \mathrm{H}), 1.78-1.72(\mathrm{~m}, 1 \mathrm{H}), 1.68-1.24(\mathrm{~m}, 8 \mathrm{H}), 1.42(\mathrm{~s}, 9 \mathrm{H}), 1.24(\mathrm{~d}, J=12.8 \mathrm{~Hz}$, $1 \mathrm{H}), 1.00(\mathrm{~s}, 3 \mathrm{H}), 0.98-0.94(\mathrm{~m}, 1 \mathrm{H}), 0.92(\mathrm{~d}, J=6.8 \mathrm{~Hz}, 3 \mathrm{H}), 0.84(\mathrm{~s}, 3 \mathrm{H}), 0.73(\mathrm{~s}, 3 \mathrm{H})$; HRMS (ESI+) $\mathrm{m} / \mathrm{z}$ Found $[\mathrm{M}+\mathrm{H}]^{+}$585.3893, $\mathrm{C}_{34} \mathrm{H}_{53} \mathrm{~N}_{2} \mathrm{O}_{6}{ }^{+}$requires 585.3898.

\section{Data for $11\left(\mathrm{R}={ }^{\mathrm{n}} \mathrm{Pr}, \mathrm{EWG}=\mathrm{CO}_{2}{ }^{\mathrm{t}} \mathrm{Bu}\right)$}

${ }^{1} \mathrm{H}-N M R\left(\mathrm{~d}_{6}\right.$-DMSO, $\left.120^{\circ} \mathrm{C}, 500 \mathrm{MHz}\right): 7.39-7.30(\mathrm{~m}, 5 \mathrm{H}), 5.17(\mathrm{~d}, J=12.4 \mathrm{~Hz}, 1 \mathrm{H}), 5.11$ (d, $J=12.4 \mathrm{~Hz}, 1 \mathrm{H}), 5.10-5.09(\mathrm{~m}, 1 \mathrm{H}), 5.08(\mathrm{~d}, J=10.9 \mathrm{~Hz}, 1 \mathrm{H}), 2.90(\mathrm{~s}, 6 \mathrm{H}), 2.54-2.46$ (m, 1H), $2.24(\mathrm{dd}, J=15.1,4.2 \mathrm{~Hz}, 1 \mathrm{H}), 2.19(\mathrm{dd}, J=15.1,6.2 \mathrm{~Hz}, 1 \mathrm{H}), 2.16-2.09(\mathrm{~m}, 2 \mathrm{H})$, 1.77-1.73 (m, 1H), 1.68-1.62 (m, 2H), 1.55-1.13 (m, 11H), $1.42(\mathrm{~s}, 9 \mathrm{H}), 1.01(\mathrm{~s}, 3 \mathrm{H}), 0.98-$ $0.93(\mathrm{~m}, 1 \mathrm{H}), 0.84(\mathrm{~s}, 3 \mathrm{H}), 0.82(\mathrm{t}, J=7.0 \mathrm{~Hz}, 3 \mathrm{H}), 0.73(\mathrm{~s}, 3 \mathrm{H})$; HRMS (ESI+) $\mathrm{m} / \mathrm{z}$ Found 
$[\mathrm{M}+\mathrm{H}]^{+}$613.4207, $\mathrm{C}_{36} \mathrm{H}_{57} \mathrm{~N}_{2} \mathrm{O}_{6}{ }^{+}$requires 613.4211.

\section{Data for $12\left(\mathrm{R}=\mathrm{i} \mathrm{Pr}, \mathrm{EWG}=\mathrm{CO}_{2}{ }^{\mathrm{t}} \mathrm{Bu}\right)$}

${ }^{1} \mathrm{H}-N M R\left(\mathrm{~d}_{6}\right.$-DMSO, $\left.120^{\circ} \mathrm{C}, 500 \mathrm{MHz}\right): 7.39-7.30(\mathrm{~m}, 5 \mathrm{H}), 5.15(\mathrm{~d}, \mathrm{~J}=12.3 \mathrm{~Hz}, 1 \mathrm{H}), 5.12$ (d, $J=12.3 \mathrm{~Hz}, 1 \mathrm{H}), 5.09(\mathrm{~d}, J=11.0 \mathrm{~Hz}, 1 \mathrm{H}), 5.08-5.06(\mathrm{~m}, 1 \mathrm{H}), 2.90(\mathrm{~s}, 6 \mathrm{H}), 2.61-2.56$ $(\mathrm{m}, 1 \mathrm{H}), 2.19(\mathrm{dd}, J=16.1,6.1 \mathrm{~Hz}, 1 \mathrm{H}), 2.14-2.05(\mathrm{~m}, 2 \mathrm{H}), 1.91$ (double septet, $J=9.4$, $6.8 \mathrm{~Hz}, 1 \mathrm{H}), 1.86(\mathrm{dd}, J=16.1,4.3 \mathrm{~Hz}, 1 \mathrm{H}), 1.77-1.60(\mathrm{~m}, 3 \mathrm{H}), 1.54-1.23(\mathrm{~m}, 7 \mathrm{H}), 1.42(\mathrm{~s}$, $9 \mathrm{H}), 1.02(\mathrm{~s}, 3 \mathrm{H}), 0.98-0.93(\mathrm{~m}, 1 \mathrm{H}), 0.87(\mathrm{~d}, J=6.9 \mathrm{~Hz}, 3 \mathrm{H}), 0.84(\mathrm{~s}, 3 \mathrm{H}), 0.81(\mathrm{~d}, J=6.8$ $\mathrm{Hz}, 3 \mathrm{H}), 0.72(\mathrm{~s}, 3 \mathrm{H})$; HRMS (ESI+) $\mathrm{m} / \mathrm{z}$ Found $\left[\mathrm{M}+\mathrm{NH}_{4}\right]^{+} 630.4470, \mathrm{C}_{36} \mathrm{H}_{60} \mathrm{~N}_{3} \mathrm{O}_{6}^{+}$requires 630.4477.

\section{Data for 13 (R=Me, EWG= $\left.\mathrm{CO}_{2} \mathrm{Et}\right)$}

${ }^{1} \mathrm{H}-N M R\left(\mathrm{~d}_{6}\right.$-DMSO, $\left.120{ }^{\circ} \mathrm{C}, 500 \mathrm{MHz}\right): 7.40-7.30(\mathrm{~m}, 5 \mathrm{H}), 5.16(\mathrm{~d}, \mathrm{~J}=12.4 \mathrm{~Hz}, 1 \mathrm{H}), 5.12$ $(\mathrm{d}, J=12.4 \mathrm{~Hz}, 1 \mathrm{H}), 5.06(\mathrm{dd}, J=11.3,2.2 \mathrm{~Hz}, 1 \mathrm{H}), 4.87(\mathrm{~d}, J=10.8 \mathrm{~Hz}, 1 \mathrm{H}), 4.07$ (dq, $J$ $=7.1,1.6 \mathrm{~Hz}, 2 \mathrm{H}), 2.89(\mathrm{~s}, 6 \mathrm{H}), 2.68-2.59(\mathrm{~m}, 1 \mathrm{H}), 2.32(\mathrm{dd}, J=14.9,3.7 \mathrm{~Hz}, 1 \mathrm{H}), 2.17-$ $2.09(\mathrm{~m}, 2 \mathrm{H}), 2.09(\mathrm{dd}, J=14.9,8.3 \mathrm{~Hz}, 1 \mathrm{H}), 1.78-1.72(\mathrm{~m}, 1 \mathrm{H}), 1.68-1.60(\mathrm{~m}, 2 \mathrm{H}), 1.54-$ $1.26(\mathrm{~m}, 6 \mathrm{H}), 1.23(\mathrm{~d}, J=12.8 \mathrm{~Hz}, 1 \mathrm{H}), 1.20(\mathrm{t}, J=7.1 \mathrm{~Hz}, 3 \mathrm{H}), 1.00(\mathrm{~s}, 3 \mathrm{H}), 0.98-0.93(\mathrm{~m}$, $1 \mathrm{H}), 0.91(\mathrm{~d}, J=6.8 \mathrm{~Hz}, 3 \mathrm{H}), 0.84(\mathrm{~s}, 3 \mathrm{H}), 0.73(\mathrm{~s}, 3 \mathrm{H})$; HRMS (ESI+) $\mathrm{m} / z$ Found $[\mathrm{M}+\mathrm{H}]^{+}$ 557.3586, $\mathrm{C}_{32} \mathrm{H}_{49} \mathrm{~N}_{2} \mathrm{O}_{6}{ }^{+}$requires 557.3585.

\section{Data for $14\left(\mathrm{R}={ }^{\mathrm{n}} \mathrm{Pr}, \mathrm{EWG}=\mathrm{CO}_{2} \mathrm{Et}\right)$}

${ }^{1} \mathrm{H}-\mathrm{NMR}\left(\mathrm{d}_{6}\right.$-DMSO, $\left.120{ }^{\circ} \mathrm{C}, 500 \mathrm{MHz}\right): 7.40-7.31(\mathrm{~m}, 5 \mathrm{H}), 5.16(\mathrm{~d}, J=12.4 \mathrm{~Hz}, 1 \mathrm{H}), 5.11$ (d, $J=12.4 \mathrm{~Hz}, 1 \mathrm{H}), 5.10(\mathrm{dd}, J=11.2,2.2 \mathrm{~Hz}, 1 \mathrm{H}), 5.05(\mathrm{~d}, J=10.6 \mathrm{~Hz}, 1 \mathrm{H}), 4.05$ (dq, J $=7.1,3.1 \mathrm{~Hz}, 2 \mathrm{H}), 2.89(\mathrm{~s}, 6 \mathrm{H}), 2.58-2.52(\mathrm{~m}, 1 \mathrm{H}), 2.29(\mathrm{dd}, J=15.3,4.4 \mathrm{~Hz}, 1 \mathrm{H}), 2.25$ $(\mathrm{dd}, J=15.3,6.1 \mathrm{~Hz}, 1 \mathrm{H}), 2.15-2.07(\mathrm{~m}, 2 \mathrm{H}), 1.78-1.73(\mathrm{~m}, 1 \mathrm{H}), 1.68-1.60(\mathrm{~m}, 2 \mathrm{H}), 1.54-$ $1.12(\mathrm{~m}, 10 \mathrm{H}), 1.23(\mathrm{~d}, J=12.8 \mathrm{~Hz}, 1 \mathrm{H}), 1.19(\mathrm{t}, J=7.1 \mathrm{~Hz}, 3 \mathrm{H}), 1.00(\mathrm{~s}, 3 \mathrm{H}), 0.98-0.93$ $(\mathrm{m}, 1 \mathrm{H}), 0.84(\mathrm{~s}, 3 \mathrm{H}), 0.81(\mathrm{t}, J=7.1 \mathrm{~Hz}, 3 \mathrm{H}), 0.73(\mathrm{~s}, 3 \mathrm{H}) ; \mathrm{HRMS}(\mathrm{ESI}+) \mathrm{m} / \mathrm{z}$ Found $[\mathrm{M}+\mathrm{H}]^{+}$585.3895, $\mathrm{C}_{34} \mathrm{H}_{53} \mathrm{~N}_{2} \mathrm{O}_{6}{ }^{+}$requires 585.3898.

\section{Data for 15 (R='Pr, EWG= $\left.\mathrm{CO}_{2} \mathrm{Et}\right)$}

${ }^{1} \mathrm{H}-\mathrm{NMR}\left(\mathrm{d}_{6}\right.$-DMSO, $\left.120{ }^{\circ} \mathrm{C}, 500 \mathrm{MHz}\right): 7.39-7.31(\mathrm{~m}, 5 \mathrm{H}), 5.15(\mathrm{~d}, J=12.4 \mathrm{~Hz}, 1 \mathrm{H}), 5.11$ (d, $J=12.4 \mathrm{~Hz}, 1 \mathrm{H}), 5.09$ (d, $J=10.9 \mathrm{~Hz}, 1 \mathrm{H}), 5.06$ (dd, $J=11.3,2.2 \mathrm{~Hz}, 1 \mathrm{H}), 4.04$ (dq, J $=7.1,2.0 \mathrm{~Hz}, 2 \mathrm{H}), 2.90(\mathrm{~s}, 6 \mathrm{H}), 2.63-2.59(\mathrm{~m}, 1 \mathrm{H}), 2.26(\mathrm{dd}, J=15.9,5.9 \mathrm{~Hz}, 1 \mathrm{H}), 2.12-$ $2.04(\mathrm{~m}, 2 \mathrm{H}), 1.94(\mathrm{dd}, J=15.9,4.7 \mathrm{~Hz}, 1 \mathrm{H}), 1.94-1.88(\mathrm{~m}, 1 \mathrm{H}), 1.76-1.72(\mathrm{~m}, 1 \mathrm{H}), 1.69-$ $1.60(\mathrm{~m}, 2 \mathrm{H}), 1.53-1.24(\mathrm{~m}, 6 \mathrm{H}), 1.24(\mathrm{~d}, J=12.8 \mathrm{~Hz}, 1 \mathrm{H}), 1.20(\mathrm{t}, J=7.1 \mathrm{~Hz}, 3 \mathrm{H}), 1.01(\mathrm{~s}$, $3 \mathrm{H}), 0.98-0.93(\mathrm{~m}, 1 \mathrm{H}), 0.86(\mathrm{~d}, J=6.9 \mathrm{~Hz}, 3 \mathrm{H}), 0.83(\mathrm{~s}, 3 \mathrm{H}), 0.79(\mathrm{~d}, J=6.8 \mathrm{~Hz}, 3 \mathrm{H}), 0.71$ $(\mathrm{s}, 3 \mathrm{H})$; HRMS (ESI+) $\mathrm{m} / \mathrm{z}$ Found $\left[\mathrm{M}+\mathrm{NH}_{4}\right]^{+} 602.4164, \mathrm{C}_{34} \mathrm{H}_{56} \mathrm{~N}_{3} \mathrm{O}_{6}{ }^{+}$requires 602.4164 .

\section{Data for 16 (R,EWG=-(0- $\left.\left.\mathrm{C}_{4} \mathrm{H}_{6}\right) \mathrm{OC}(\mathrm{O})-\right)$}

${ }^{1} \mathrm{H}-\mathrm{NMR}\left(\mathrm{d}_{6}\right.$-DMSO, $\left.120{ }^{\circ} \mathrm{C}, 500 \mathrm{MHz}\right): 7.36-7.20(\mathrm{~m}, 7 \mathrm{H}), 7.05(\mathrm{dt}, J=7.5,1.1 \mathrm{~Hz}, 1 \mathrm{H})$, 6.95 (dd, $J=8.0,1.1 \mathrm{~Hz}, 1 \mathrm{H}), 5.39$ (dd, $J=11.5,2.1 \mathrm{~Hz}, 1 \mathrm{H}), 4.91(\mathrm{~d}, J=12.3 \mathrm{~Hz}, 1 \mathrm{H})$, $4.87(\mathrm{~d}, J=9.3 \mathrm{~Hz}, 1 \mathrm{H}), 4.66(\mathrm{~d}, J=12.3 \mathrm{~Hz}, 1 \mathrm{H}), 3.79$ (ddd, $J=9.3,6.7,2.6 \mathrm{~Hz}, 1 \mathrm{H}$ ), $2.92(\mathrm{dd}, J=16.6,6.7 \mathrm{~Hz}, 1 \mathrm{H}), 2.83(\mathrm{~s}, 6 \mathrm{H}), 2.82(\mathrm{dd}, J=16.6,2.6 \mathrm{~Hz}, 1 \mathrm{H}), 2.22(\mathrm{ddt}, J=$ 12.5, 11.9, 4.0 Hz, $1 \mathrm{H}), 2.16(\mathrm{dt}, J=12.8,3.6 \mathrm{~Hz}, 1 \mathrm{H}), 1.91-1.86(\mathrm{~m}, 1 \mathrm{H}), 1.81-1.78(\mathrm{~m}$, $1 \mathrm{H}), 1.66-1.59(\mathrm{~m}, 2 \mathrm{H}), 1.45-1.27(\mathrm{~m}, 4 \mathrm{H}), 1.23(\mathrm{~d}, J=12.8 \mathrm{~Hz}, 1 \mathrm{H}), 1.10-0.94(\mathrm{~m}, 1 \mathrm{H})$, $0.93(\mathrm{~s}, 3 \mathrm{H}), 0.83(\mathrm{~s}, 3 \mathrm{H}), 0.73(\mathrm{~s}, 3 \mathrm{H})$; HRMS (ESI+) $\mathrm{m} / \mathrm{z}$ Found $[\mathrm{M}+\mathrm{Na}]^{+}$611.3118, $\mathrm{C}_{35} \mathrm{H}_{44} \mathrm{~N}_{2} \mathrm{O}_{6} \mathrm{Na}^{+}$requires 611.3092.

\section{Data for 17 (R=Ph, EWG=COPh)}


${ }^{1} \mathrm{H}-\mathrm{NMR}\left(\mathrm{d}_{6}-\mathrm{DMSO}, 120{ }^{\circ} \mathrm{C}, 500 \mathrm{MHz}\right): 7.81(\mathrm{~d}, J=7.6 \mathrm{~Hz}, 2 \mathrm{H}), 7.54(\mathrm{t}, J=7.6 \mathrm{~Hz}, 1 \mathrm{H})$, $7.43(\mathrm{t}, J=7.6 \mathrm{~Hz}, 2 \mathrm{H}), 7.34-7.19(\mathrm{~m}, 7 \mathrm{H}), 7.16-7.07(\mathrm{~m}, 3 \mathrm{H}), 5.50(\mathrm{~d}, J=10.6 \mathrm{~Hz}, 1 \mathrm{H})$, $5.16(\mathrm{dd}, J=11.6,1.9 \mathrm{~Hz}, 1 \mathrm{H}), 5.02(\mathrm{~d}, J=12.3 \mathrm{~Hz}, 1 \mathrm{H}), 4.77(\mathrm{~d}, J=12.3 \mathrm{~Hz}, 1 \mathrm{H}), 4.21$ (ddd, $J=10.6,9.5,3.6 \mathrm{~Hz}, 1 \mathrm{H}), 3.32(\mathrm{dd}, J=15.9,9.5 \mathrm{~Hz}, 1 \mathrm{H}), 3.22(\mathrm{dd}, J=15.9,3.6 \mathrm{~Hz}$, $1 \mathrm{H}), 2.97(\mathrm{~s}, 6 \mathrm{H}), 2.37$ (ddt, $J=12.2,11.9,4.1 \mathrm{~Hz}, 1 \mathrm{H}), 2.06(\mathrm{dt}, J=12.8,3.3 \mathrm{~Hz}, 1 \mathrm{H}$ ), 1.83-1.58 (m, 4H), 1.50-1.21 (m, 5H), $1.33(\mathrm{~d}, J=12.8 \mathrm{~Hz}, 1 \mathrm{H}), 0.96-0.90(\mathrm{~m}, 1 \mathrm{H}), 0.88(\mathrm{~s}$, $3 \mathrm{H}), 0.81(\mathrm{~s}, 3 \mathrm{H}), 0.73(\mathrm{~s}, 3 \mathrm{H})$; HRMS (ESI+) $\mathrm{m} / z$ Found $[\mathrm{M}+\mathrm{H}]^{+} 651.3797, \mathrm{C}_{41} \mathrm{H}_{51} \mathrm{~N}_{2} \mathrm{O}_{5}{ }^{+}$ requires 651.3792 .

\section{Acid hydrolysis of 13}<smiles>CCOCCC(C)C(NC(C)=O)C(=O)OCC</smiles>

18

A solution of 13 (105 mg, $0.19 \mathrm{mmol})$ in TFA/ $\mathrm{H}_{2} \mathrm{O}(9: 1,3 \mathrm{ml})$ was stirred at ambient temperature for 6 hours before being quenched with saturated aqueous $\mathrm{NaHCO}_{3}(15 \mathrm{ml})$ and extracted with DCM $(3 \times 15 \mathrm{ml})$ and EtOAc $(3 \times 50 \mathrm{ml})$. The combined organics were concentrated in vacuo to give an orange oil/solid which was purified by column chromatography (silica, petroleum ether $\left(40-60{ }^{\circ} \mathrm{C}\right): \mathrm{Et}_{2} \mathrm{O}, 1: 1$ gradient to neat $\left.\mathrm{Et}_{2} \mathrm{O}\right)$ to give 18 as a pale yellow oil $(40.4 \mathrm{mg}, 0.12 \mathrm{mmol}, 61 \%)$. ${ }^{1} \mathrm{H}-\mathrm{NMR}\left(\mathrm{CDCl}_{3}, 27{ }^{\circ} \mathrm{C}, 500 \mathrm{MHz}\right)$ : 7.39-7.30 (m, 5H), $5.78(\mathrm{~d}, J=8.4 \mathrm{~Hz}, 1 \mathrm{H}), 5.11(\mathrm{~d}, J=12.6 \mathrm{~Hz}, 1 \mathrm{H}), 5.08(\mathrm{~d}, J=12.6 \mathrm{~Hz}$, $1 \mathrm{H}), 4.89$ (dd, $J=8.4,3.0 \mathrm{~Hz}, 1 \mathrm{H}), 4.22-4.15(\mathrm{~m}, 2 \mathrm{H}), 3.22(\mathrm{~s}, 3 \mathrm{H}), 2.98(\mathrm{~s}, 3 \mathrm{H}), 2.50$ (dd, $J=16.9,8.8 \mathrm{~Hz}, 1 \mathrm{H}), 2.41-2.33(\mathrm{~m}, 1 \mathrm{H}), 2.21(\mathrm{dd}, J=16.9,5.2 \mathrm{~Hz}, 1 \mathrm{H}), 1.29(\mathrm{t}, J=7.1$ $\mathrm{Hz}, 3 \mathrm{H}), 0.87(\mathrm{~d}, \mathrm{~J}=6.9 \mathrm{~Hz}, 3 \mathrm{H})$; HRMS (ESI+) $\mathrm{m} / \mathrm{z}$ Found $[\mathrm{M}+\mathrm{H}]^{+} 351.1911, \mathrm{C}_{18} \mathrm{H}_{27} \mathrm{~N}_{2} \mathrm{O}_{5}{ }^{+}$ requires 351.1914 .

\section{Preparation of the pyroglutamic acid derivative 19}<smiles>CCOC(=O)CC(C)[C@H](NC(C)=O)C(=O)N[C@H](C(C)=O)C(=O)NC(C)=O</smiles>

18

19

To a solution of 18 (25 mg, $0.07 \mathrm{mmol})$ in EtOAc $(2.5 \mathrm{ml})$ was added $\mathrm{Pd} / \mathrm{C}(10 \%$ by mass, $2.5 \mathrm{mg}$ ) and the mixture stirred under an atmosphere of hydrogen (approx. 1 bar) for 16 hours. The reaction mixture was filtered through celite and concentrated in vacuo to give a yellow oil which was re-dissolved in $\mathrm{MeOH}(2.5 \mathrm{ml})$ and heated at reflux under argon for 2 days. The solution was concentrated in vacuo to give essentially clean 19 as a pale yellow oil (10.2 mg, $0.06 \mathrm{mmol}, 86 \%)$. A small sample was purified for analysis by column chromatography (silica, $\mathrm{MeOH}$ in $\mathrm{DCM}, 1 \%$ gradient to $10 \%) .{ }^{1} \mathrm{H}-\mathrm{NMR}\left(\mathrm{CDCl}_{3}, 27^{\circ} \mathrm{C}, 500\right.$ $\mathrm{MHz}): 5.93(\mathrm{bs}, 1 \mathrm{H}), 4.61(\mathrm{~d}, J=6.9 \mathrm{~Hz}, 1 \mathrm{H}), 3.06(\mathrm{~s}, 3 \mathrm{H}), 3.02(\mathrm{~s}, 3 \mathrm{H}), 2.86$ (dddq, $J=$ 8.0, 7.1, 6.9, 3.7 Hz, 1H), 2.62 (dd, $J=16.5,8.0 \mathrm{~Hz}, 1 \mathrm{H}), 2.10$ (dd, $J=16.5,3.7 \mathrm{~Hz}, 1 \mathrm{H}$ ), $1.00(\mathrm{~d}, \mathrm{~J}=7.1 \mathrm{~Hz}, 3 \mathrm{H})$; HRMS (ESI+) $\mathrm{m} / z$ Found $[\mathrm{M}+\mathrm{H}]^{+} 171.1131, \mathrm{C}_{8} \mathrm{H}_{15} \mathrm{~N}_{2} \mathrm{O}_{3}{ }^{+}$requires 171.1128. 


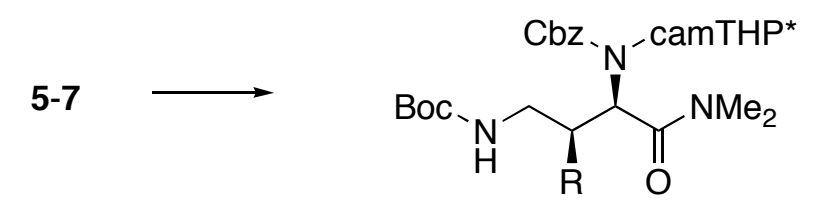

20-22

To a stirred solution of the nitro compound $(5-7,0.05 \mathrm{mmol})$ in THF $(0.5 \mathrm{ml})$ and $\mathrm{MeOH}$ $(0.3 \mathrm{ml})$ at ambient temperature under argon was added $\mathrm{NiCl}_{2} \cdot 6 \mathrm{H}_{2} \mathrm{O}(24 \mathrm{mg}, 0.1 \mathrm{mmol})$. After 10 minutes the yellow solution was cooled to $0{ }^{\circ} \mathrm{C}$ and $\mathrm{NaBH}_{4}(12 \mathrm{mg}, 0.3 \mathrm{mmol})$ added and the resultant dark green solution stirred for 3 hours at $0{ }^{\circ} \mathrm{C}$ before the addition of $\mathrm{Boc}_{2} \mathrm{O}$ ( $33 \mathrm{mg}, 0.15 \mathrm{mmol}$ ). The reaction was allowed to warm to room temperature and stirred under argon for a further 16 hours before being filtered through silica (10 mm x 10 $\mathrm{mm}$ dia.) eluting with $\mathrm{Et}_{2} \mathrm{O}(20 \mathrm{ml})$ and concentrated in vacuo to give a pale yellow oil. The crude product was purified by column chromatography (silica, petroleum ether (40-60 $\left.{ }^{\circ} \mathrm{C}\right)$ : $\mathrm{Et}_{2} \mathrm{O}, 3: 1$ gradient to $\left.1: 3\right)$.

\section{Data for $20(\mathrm{R}=\mathrm{Ph})$}

${ }^{1} \mathrm{H}-N M R\left(\mathrm{~d}_{6}\right.$-DMSO, $\left.120^{\circ} \mathrm{C}, 500 \mathrm{MHz}\right): 7.35-7.14(\mathrm{~m}, 10 \mathrm{H}), 5.49$ (bs, $\left.1 \mathrm{H}\right), 5.34(\mathrm{~d}, J=10.5$ $\mathrm{Hz}, 1 \mathrm{H}), 5.07(\mathrm{dd}, J=11.5,1.9 \mathrm{~Hz}, 1 \mathrm{H}), 5.06(\mathrm{~d}, J=12.3 \mathrm{~Hz}, 1 \mathrm{H}), 4.84(\mathrm{~d}, J=12.3 \mathrm{~Hz}$, $1 \mathrm{H}$ ), $3.81(\mathrm{dt}, J=10.5,6.1 \mathrm{~Hz}, 1 \mathrm{H}$ ), $3.19(\mathrm{t}, J=6.1 \mathrm{~Hz}, 2 \mathrm{H}), 2.95(\mathrm{~s}, 6 \mathrm{H}), 2.30$ (ddt, $J=$ 12.3, 11.9, $4.0 \mathrm{~Hz}, 1 \mathrm{H}), 2.03(\mathrm{dt}, J=9.4,3.6 \mathrm{~Hz}, 1 \mathrm{H}), 1.78-1.74(\mathrm{~m}, 1 \mathrm{H}), 1.63-1.56(\mathrm{~m}$, $1 \mathrm{H}), 1.50-1.47(\mathrm{~m}, 1 \mathrm{H}), 1.44-1.19(\mathrm{~m}, 6 \mathrm{H}), 1.32(\mathrm{~s}, 9 \mathrm{H}), 1.09(\mathrm{~d}, J=12.7,1 \mathrm{H}), 0.93-0.88$ $(\mathrm{m}, 1 \mathrm{H}), 0.87(\mathrm{~s}, 3 \mathrm{H}), 0.80(\mathrm{~s}, 3 \mathrm{H}), 0.71(\mathrm{~s}, 3 \mathrm{H})$; HRMS (ESI+) $\mathrm{m} / \mathrm{z}$ Found $[\mathrm{M}+\mathrm{H}]^{+}$ $662.4163, \mathrm{C}_{39} \mathrm{H}_{56} \mathrm{~N}_{3} \mathrm{O}_{6}{ }^{+}$requires 662.4164 .

\section{Data for 21 ( $R=2-N a p)$}

${ }^{1} \mathrm{H}-N M R\left(\mathrm{~d}_{6}\right.$-DMSO, $\left.120{ }^{\circ} \mathrm{C}, 500 \mathrm{MHz}\right): 7.82(\mathrm{dt}, J=9.4,3.3 \mathrm{~Hz}, 1 \mathrm{H}), 7.76-7.73(\mathrm{~m}, 2 \mathrm{H})$, $7.70(\mathrm{~d}, J=8.5 \mathrm{~Hz}, 1 \mathrm{H}), 7.46-7.43(\mathrm{~m}, 3 \mathrm{H}), 7.28-7.2(\mathrm{~m}, 3 \mathrm{H}), 7.12(\mathrm{~d}, J=7.1 \mathrm{~Hz}, 2 \mathrm{H}), 5.62$ (bs, $1 \mathrm{H}), 5.49(\mathrm{~d}, J=10.7 \mathrm{~Hz}, 1 \mathrm{H}), 5.03(\mathrm{dd}, J=11.6,2.0 \mathrm{~Hz}, 1 \mathrm{H}), 5.01(\mathrm{~d}, J=12.2 \mathrm{~Hz}$, $1 \mathrm{H}), 4.79(\mathrm{~d}, J=12.2 \mathrm{~Hz}, 1 \mathrm{H}), 4.01(\mathrm{dt}, J=10.7,6.0 \mathrm{~Hz}, 1 \mathrm{H}), 3.29-3.26(\mathrm{~m}, 2 \mathrm{H}), 2.98(\mathrm{~s}$, $6 \mathrm{H}), 2.35$ (ddt, $J=12.2,11.9,4.0 \mathrm{~Hz}, 1 \mathrm{H}), 1.98(\mathrm{dt}, J=12.5,3.0 \mathrm{~Hz}, 1 \mathrm{H}), 1.79-1.75(\mathrm{~m}$, $1 \mathrm{H}), 1.61-1.51(\mathrm{~m}, 3 \mathrm{H}), 1.42-1.19(\mathrm{~m}, 5 \mathrm{H}), 1.26(\mathrm{~s}, 9 \mathrm{H}), 1.06(\mathrm{~d}, J=12.7,1 \mathrm{H}), 0.91-0.85$ $(\mathrm{m}, 1 \mathrm{H}), 0.82(\mathrm{~s}, 3 \mathrm{H}), 0.78(\mathrm{~s}, 3 \mathrm{H}), 0.71(\mathrm{~s}, 3 \mathrm{H})$; HRMS (ESI+) $\mathrm{m} / \mathrm{z}$ Found $\left[\mathrm{M}+\mathrm{NH}_{4}\right]^{+}$ 729.4592, $\mathrm{C}_{43} \mathrm{H}_{61} \mathrm{~N}_{4} \mathrm{O}_{6}{ }^{+}$requires 729.4586 .

\section{Data for 22 (R=PMP)}

${ }^{1} \mathrm{H}-N M R\left(\mathrm{~d}_{6}\right.$-DMSO, $\left.120{ }^{\circ} \mathrm{C}, 500 \mathrm{MHz}\right)$ : 7.35-7.31 (m, 3H), 7.25-7.23 (m, $\left.2 \mathrm{H}\right), 7.17-7.14$ $(\mathrm{m}, 2 \mathrm{H}), 6.76-6.73(\mathrm{~m}, 2 \mathrm{H}), 5.44(\mathrm{bs}, 1 \mathrm{H}), 5.29(\mathrm{~d}, J=10.7 \mathrm{~Hz}, 1 \mathrm{H}), 5.09(\mathrm{~d}, J=12.3 \mathrm{~Hz}$, $1 \mathrm{H}), 5.08$ (dd, $J=11.4,2.0 \mathrm{~Hz}, 1 \mathrm{H}), 4.86(\mathrm{~d}, J=12.3 \mathrm{~Hz}, 1 \mathrm{H}), 3.75(\mathrm{dt}, J=10.6,6.3 \mathrm{~Hz}$, $1 \mathrm{H}), 3.74(\mathrm{~s}, 3 \mathrm{H}), 3.16-3.13(\mathrm{~m}, 2 \mathrm{H}), 2.95(\mathrm{~s}, 6 \mathrm{H}), 2.29(\mathrm{ddt}, J=12.3,11.9,4.1 \mathrm{~Hz}, 1 \mathrm{H})$, 2.03 (dt, $J=12.7,3.2 \mathrm{~Hz}, 1 \mathrm{H}), 1.78-1.74(\mathrm{~m}, 1 \mathrm{H}), 1.62-1.57(\mathrm{~m}, 2 \mathrm{H}), 1.49-1.20(\mathrm{~m}, 6 \mathrm{H})$, $1.33(\mathrm{~s}, 9 \mathrm{H}), 1.09(\mathrm{~d}, J=12.7,1 \mathrm{H}), 0.93-0.88(\mathrm{~m}, 1 \mathrm{H}), 0.88(\mathrm{~s}, 3 \mathrm{H}), 0.80(\mathrm{~s}, 3 \mathrm{H}), 0.71(\mathrm{~s}$, $3 \mathrm{H})$; HRMS (ESI+) $\mathrm{m} / \mathrm{z}$ Found $[\mathrm{M}+\mathrm{H}]^{+} 692.4283, \mathrm{C}_{40} \mathrm{H}_{58} \mathrm{~N}_{3} \mathrm{O}_{7}^{+}$requires 692.4275 . 